OPEN

SUBJECT AREAS:

MATERIALS SCIENCE

OPTICAL DATA STORAGE

OPTICAL PHYSICS

APPLIED PHYSICS

Received

28 June 2013

Accepted

26 September 2013

Published

16 October 2013

Correspondence and requests for materials should be addressed to

R.J.H. (R.J.Hicken@ exeter.ac.uk)

\section{Observation of $\mathrm{T}_{2}$-like coherent optical phonons in epitaxial $\mathrm{Ge}_{2} \mathrm{Sb}_{2} \mathrm{Te}_{5}$ / GaSb(001) films}

\author{
A. Shalini' ${ }^{1}$, Y. Liu' , U.A.S. Al-Jarah', G. P. Srivastava' ', C. D. Wright ${ }^{2}$, F. Katmis ${ }^{3}$, W. Braun ${ }^{3,4}$ \& R. J. Hicken'
}

'School of Physics and Astronomy, University of Exeter, Stocker Road, Exeter, EX4 4QL, UK, ${ }^{2}$ School of Engineering, Computing and Mathematics, University of Exeter, North Park Road, Exeter, EX4 4QF, UK, ${ }^{3}$ Paul Drude Institute for Solid State Electronics, Hausvogteiplatz 5-7, 10117 Berlin, Germany, ${ }^{4}$ Createc Fischer \& Co. GmbH, Industriestr. 9, 74391 Erligheim, Germany.

The phonon spectrum of $\mathrm{Ge}_{2} \mathrm{Sb}_{2} \mathrm{Te}_{5}$ is a signature of its crystallographic structure and underlies the phase transition process used in memory applications. Epitaxial materials allow coherent optical phonons to be studied in femtosecond anisotropic reflectance measurements. A dominant phonon mode with frequency of 3.4 THz has been observed in epitaxial $\mathrm{Ge}_{2} \mathrm{Sb}_{2} \mathrm{Te}_{5}$ grown on $\mathrm{GaSb}(001)$. The dependence of signal strength upon pump and probe polarization is described by a theory of transient stimulated Raman scattering that accounts for the symmetry of the crystallographic structure through use of the Raman tensor. The $3.4 \mathrm{THz}$ mode has the character of the 3 dimensional $\mathrm{T}_{2}$ mode expected for the $\mathrm{O}_{\mathrm{h}}$ point group, confirming that the underlying crystallographic structure is cubic. New modes are observed in both $\mathrm{Ge}_{2} \mathrm{Sb}_{2} \mathrm{Te}_{5}$ and $\mathrm{GaSb}$ after application of large pump fluences, and are interpreted as 1 and 2 dimensional modes associated with segregation of $\mathrm{Sb}$.

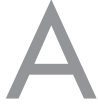

lloys such as $\mathrm{Ge}_{2} \mathrm{Sb}_{2} \mathrm{Te}_{5}$ have attracted great interest due to their favorable properties for phase change memory applications ${ }^{1}$. The use of $\mathrm{Ge}_{2} \mathrm{Sb}_{2} \mathrm{Te}_{5}$ for optical disk coatings is well established while more recently $\mathrm{Ge}_{2} \mathrm{Sb}_{2} \mathrm{Te}_{5}$ has also been used in the development of phase change random access memory ${ }^{2}$ and within new paradigms for bio-inspired computing ${ }^{3,4}$. The popularity of $\mathrm{Ge}_{2} \mathrm{Sb}_{2} \mathrm{Te}_{5}$ stems from the fast and reversible phase transition that occurs between its amorphous and cubic crystalline phases, and the strong contrast in electrical conductance and optical reflectance that these phases exhibit. The cubic phase has a distorted rock-salt structure with a high density of vacancies. The distribution of vacancies and hence the bonding within the material, and its modification during the phase transition, remains poorly understood. Knowledge and understanding of the phonon spectrum can provide a fingerprint of the crystallographic structure while large amplitude phonons may appear as a precursor to the phase transition itself. Although several studies of coherent optical phonon (COP) dynamics have been performed upon both amorphous and polycrystalline $\mathrm{Ge}_{2} \mathrm{Sb}_{2} \mathrm{Te}_{5}{ }^{5-9}$, no such study has been conducted on a $\mathrm{Ge}_{2} \mathrm{Sb}_{2} \mathrm{Te}_{5}$ single crystal. Therefore the growth of epitaxial $\mathrm{Ge}_{2} \mathrm{Sb}_{2} \mathrm{Te}_{5}$ films upon $\mathrm{GaSb}(001)$ substrates ${ }^{10}$ by molecular beam epitaxy provides an opportunity to explore the character of the phonon spectrum of $\mathrm{Ge}_{2} \mathrm{Sb}_{2} \mathrm{Te}_{5}$.

Time-resolved optical pump-probe measurements can provide information about both the ultrafast relaxation of photo-excited carriers and the phase and amplitude of $\mathrm{COPs}^{11,12}$. By varying the polarization of the pump and probe beams relative to the crystallographic axes, the symmetry of a particular COP can be deduced, and insight can be gained into the non-thermal nature of phonon excitation and the structural phase transition at large fluence. In this article, we present time resolved reflectance (R) and anisotropic reflectance (AR) measurements of $\mathrm{GaSb}(001)$ and $\mathrm{Ge}_{2} \mathrm{Sb}_{2} \mathrm{Te}_{5} / \mathrm{GaSb}(001)$. The results are interpreted in terms of a theory of transient stimulated Raman scattering ${ }^{11}$ that can account for the excitation of phonons by either impulsive stimulated Raman scattering (ISRS), which can be considered to include displacive excitation of coherent phonons, or the action of an optically induced space charge (SC) field ${ }^{13}$. The dependence of the signals upon the pump and probe polarization is accounted for through use of the Raman tensor. The dominant mode observed in AR measurements of $\mathrm{Ge}_{2} \mathrm{Sb}_{2} \mathrm{Te}_{5}$ will be shown to be the $\mathrm{T}_{2}$-like mode of the cubic structure. New modes observed after the application of large pump fluences are interpreted as 1 and 2 dimensional modes of $\mathrm{Sb}$, while a longer-lived transient AR signal is explained in terms of the piezoelectric response of the GaSb to a SC field. 


\section{Results}

The transient reflectance (R) response of an epitaxial $\mathrm{Ge}_{2} \mathrm{Sb}_{2} \mathrm{Te}_{5}$ $(15 \mathrm{~nm}) / \mathrm{GaSb}(001)$ structure, in which the [100] axes of the $\mathrm{Ge}_{2} \mathrm{Sb}_{2} \mathrm{Te}_{5}$ and $\mathrm{GaSb}$ are parallel is shown in Fig. 1(a) and is similar to that observed in GaSb(001) (shown in Supplementary Information S1). The red curve is a fit to a phenomenological fitting function. The procedure for fitting the $\mathrm{R}$ and AR scans is described within Supplementary Information S2. The signal is plotted on three different time scales so as to reveal all of its principal features. The initial rise of the signal occurs within about $200 \mathrm{fs}$ and is associated with photoexcitation and thermalization of an electron-hole plasma. Weak oscillations with frequency of $4.2-4.5 \mathrm{THz}$ are observed until about 1 ps time delay. The signal then changes sign after about 6 ps before reaching a minimum after about 70 ps. This region is well fitted by two exponential functions with relaxation times of $2.0 \pm$ $0.1 \mathrm{ps}$ and $13.1 \pm 0.5 \mathrm{ps}$, which can be attributed to the interaction of the electrons with incoherent optical and acoustic phonons respectively $^{14,15}$. Weak superimposed oscillations with frequency of $43 \mathrm{GHz}$ are observed until about 150 ps and can be attributed to longitudinal coherent acoustic phonons propagating normal to the plane of the sample. The final relaxation towards the ambient state is well fitted by two exponential terms. The first has relaxation time of $0.42 \pm$ $0.05 \mathrm{~ns}$ and can be assigned to carrier diffusion from the pumped spot. A longer time of $5.4 \pm 0.5 \mathrm{~ns}$ can be associated with thermal conduction within the lattice. Similar relaxation behavior has been observed previously in $\mathrm{Si}^{16,17}$. The form of the transient $\mathrm{R}$ response was found to be independent of the pump and probe polarization except for a small variation in amplitude close to zero time delay. The similarity of the $\mathrm{R}$ signals obtained from the $\mathrm{Ge}_{2} \mathrm{Sb}_{2} \mathrm{Te}_{5} / \mathrm{GaSb}$ and $\mathrm{GaSb}$ samples suggests that the signals are dominated by the response of the homo-epitaxial $\mathrm{GaSb}$ layer and substrate.

The AR response of the $\mathrm{Ge}_{2} \mathrm{Sb}_{2} \mathrm{Te}_{5} / \mathrm{GaSb}(001)$ structure is shown in Fig. 1(b) for the pump and probe electric fields parallel to the [110] and [100] axes respectively. The signal contains a large peak centred at zero time delay that lies within the temporal overlap of the pump

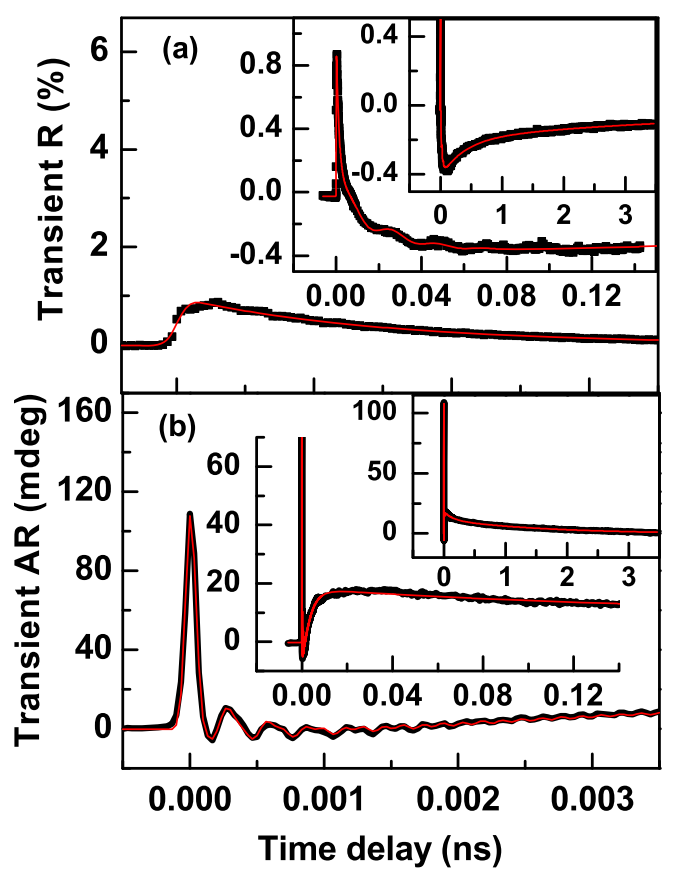

Figure 1 | Typical time resolved (a) $\mathrm{R}$ and (b) AR signals for $\mathrm{Ge}_{2} \mathrm{Sb}_{2} \mathrm{Te}_{5} /$ $\mathrm{GaSb}(001)$ with the pump and probe electric fields aligned parallel to the [110] and [100] axes respectively. The solid points represent the experimental data while the red/lines are the fitted curves described within the main text. and probe pulses, two oscillatory components of 6.7 and $3.4 \mathrm{THz}$ frequency superimposed on a multi-exponential background, and a slower (ns scale) relaxation. The initial peak arises from the specular optical Kerr effect ${ }^{18}$, an optically induced birefringence, and has maximum amplitude when the pump and probe electric fields lie $45^{\circ}$ apart. The peak occurs because the pump modifies the electron momentum distribution within the surface of the sample and is short-lived due to the small momentum relaxation time ${ }^{19}$. The multi-exponential background is fitted well by the sum of three terms with relaxation times of $500 \mathrm{fs}, 3.0 \mathrm{ps}$, and $137 \mathrm{ps}$. These terms may be associated with the momentum relaxation of charge carriers, and incoherent optical and acoustic phonons respectively ${ }^{20}$. The slowest relaxation time of $1.6 \mathrm{~ns}$ is associated with relaxation of strain in the $\mathrm{GaSb}$ that results from its piezolelectric response to a SC field. Oscillations of $\mathrm{THz}$ frequency have been widely observed in $\mathrm{R}$ measurements conducted on other materials ${ }^{12,21-25}$. If the fitted initial peak and multi-exponential background are subtracted from the AR data then the residual is well described by the sum of two damped sinusoids $(i=1,2)$ of the form $A_{i} \exp \left(-t / \tau_{i}\right) \cos \left(2 \pi f_{i} t+\phi_{i}\right)$, allowing the mode amplitudes $A_{i}$, frequencies $f_{i}$, relaxation times $\tau_{i}$, and phases $\phi_{i}$, to be determined.

AR measurements made upon the reference GaSb structure revealed largely similar features (shown in Supplementary Information S1). However the $3.4 \mathrm{THz}$ mode was observed only in the $\mathrm{Ge}_{2} \mathrm{Sb}_{2} \mathrm{Te}_{5} / \mathrm{GaSb}$ structure, confirming that it originates in the $\mathrm{Ge}_{2} \mathrm{Sb}_{2} \mathrm{Te}_{5}$ layer. Similar relaxation times were obtained for the multiexponential background and long relaxation terms, albeit with some difference in the amplitudes.

Further measurements were made as the planes of polarization of the pump and probe beams were varied relative to the crystallographic axes of the sample. Figure 2 shows typical oscillatory components in panel (a), and the variation of their frequency, amplitude and phase in panels (b-e), for the GaSb structure. The variation of the amplitude of the ns transient in the AR response is presented in the Supplementary Information S3. The angles $\varphi$ and $\theta$ used to define the orientation of the pump and probe electric fields relative to the [100] axis are shown within the inset to panel (b). From panels (b) and (d) the frequency is seen to have an essentially constant value of 6.77 THz. From panels (a) and (c) the oscillations are seen to almost vanish when the probe electric field lies along $\langle 110\rangle$ and have maximum amplitude when it lies parallel to $<100>$. The variation of amplitude and phase in (c) and (b) respectively suggests an overall $\cos (2 \theta)$ dependence upon probe polarization. Panels (d) and (e) reveal a more complicated dependence upon the pump polarization. The amplitude is a minimum when the pump electric field lies parallel to $\langle 100\rangle$ but has different values when it is parallel to [110] and $[-110]$. The range of phase of about 2 rad suggests contributions from different excitation mechanisms of different relative phase. However, when the amplitude and phase data of panels (e) and (d) respectively are plotted in the Argand plane, in panel (f), the data points are found to lie on a straight line.

\section{Discussion}

GaSb has the zincblende structure with $\mathrm{T}_{\mathrm{d}}$ point group symmetry and $A_{1}, A_{2}, E, T_{1}$ and $T_{2}$ irreducible representations at the zone centre $^{26,27}$. Of the associated phonon modes only the $T_{2}$ mode is Raman active. At the center of the Brillouin zone, the three dimensional mode splits into a non-degenerate longitudinal optical (LO) phonon and doubly degenerate transverse optical (TO) phonons with slightly lower frequency ${ }^{28}$. Raman scattering studies ${ }^{29}$ have observed the LO phonon at $7.08 \mathrm{THz}$ while neutron scattering ${ }^{30}$ measurements observe both LO and TO modes and suggest that the TO phonon is detected in the present experiments.

A theory of transient stimulated Raman scattering ${ }^{11}$ has been applied to the interpretation of the experimental results (see Supplementary Information S4). In this theory the amplitude of 

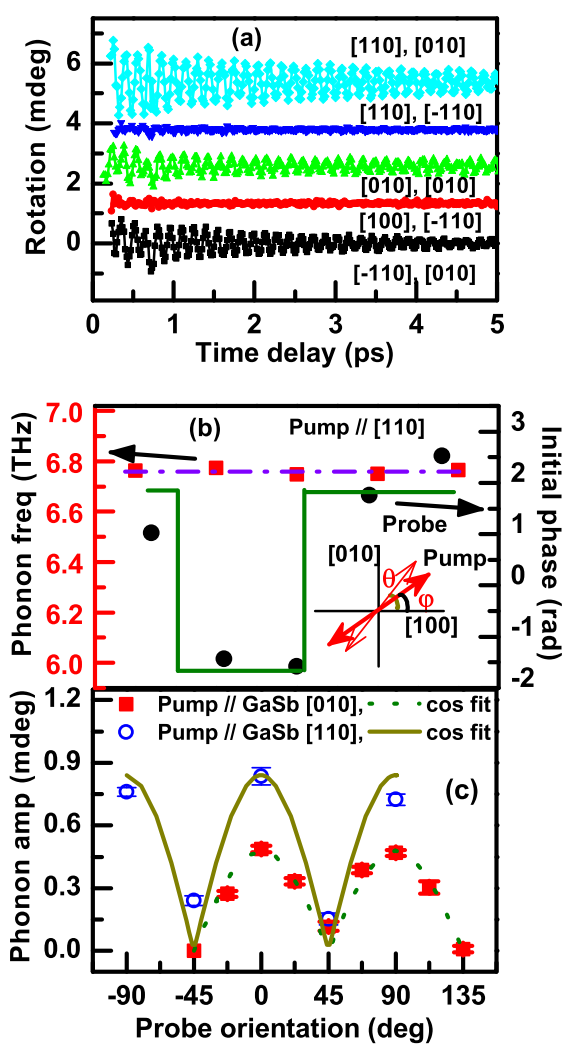

Figure $2 \mid$ Dependence of frequency, amplitude and phase of phonon oscillations in GaSb upon the orientation of the pump and probe electric fields. (a) Typical oscillatory signals after subtraction of the initial peak and multi-exponential background (Labels represent the orientation of pump and probe polarization from $\mathrm{GaSb}[100]$ axis respectively). (b) Frequency and phase, and (c) amplitude, are plotted as the probe polarization is varied. (d) Frequency and phase, and (e) amplitude, are plotted as the pump polarization is varied. The angles $\varphi$ and $\theta$ used to define the orientation of the pump and probe electric fields are shown within the inset to (b). (f) Data from (d) and (e) are plotted in the Argand plane. The purple straight line fit in (f) has been used to generate the continuous curves in $(\mathrm{d})$ and $(\mathrm{e})$.

the COP excited by ISRS is proportional to a contraction of the relevant Raman tensor with the pump electric field. Then the probe beam is resolved into two orthogonally polarized components and the Raman tensor is contracted with each in turn. The transient $\mathrm{R}$ and AR signals are proportional to the sum and difference of these contractions respectively, and also to the phonon amplitude. Using the well-known form of the Raman tensor for the 5 irreducible representations, the theory predicts that only the $A_{1}, A_{2}$ and $E$ COPs should be observed in the transient $\mathrm{R}$ measurements. For transient $\mathrm{AR}$ measurements only the $\mathrm{T}_{2 \mathrm{z}}$ mode with atomic displacement parallel to the [001] axis should appear, with amplitude proportional to $\cos (2 \theta) \sin (2 \varphi)$. A similar expression may be obtained from a simple model that considers the orientation of the pump and probe electric fields relative to inequivalent bonds within the zincblende structure (see Supplementary Information S5).

While the $\cos (2 \theta) \sin (2 \varphi)$ form describes observations of the $\mathrm{T}_{2 \mathrm{z}}$ COP in $\mathrm{Ge}(001)^{23}$, it does not fully account for the dependence on pump polarization observed in Fig. 2(d-f). Studies of COPs in GaAs ${ }^{12,24}$ have shown that the $\mathrm{T}_{2 \mathrm{z}}$ mode may also be excited by an optically induced SC field. Here photo-excited electrons and holes become spatially separated, either due to diffusion and because of their different mobilities in the photo-Dember effect ${ }^{31}$, or due to drift in opposite directions under the influence of the built-in electric field near an interface ${ }^{13}$. The latter mechanism seems more likely in the present case but both mechanisms generate an additional space charge field on sub-ps timescales. In a polar compound such as $\mathrm{GaAs}$ or $\mathrm{GaSb}$, the oppositely charged basis ions experience an impulse in opposite directions normal to the plane of the film. This leads to an oscillatory motion of $\mathrm{T}_{2 \mathrm{z}}$ character superimposed upon an offset of the basis atom positions that gives rise to the long-lived transient within the AR response (see Supplementary Information S7). The amplitude of the $\mathrm{T}_{2 \mathrm{z}} \mathrm{COP}$ excited by the SC field again exhibits a $\cos (2 \theta)$ dependence upon the probe polarization but is independent of the pump polarization.

ISRS and the SC field are expected to excite COPs of different phase. In ISRS the pump selectively excites bonds of different orientation, providing the driving force for the $\mathrm{T}_{2 \mathrm{z}}$ mode and leading to an electron-hole distribution that is anisotropic in $\mathrm{k}$-space. While the bonding will be weakened on the timescale of the electron-hole recombination time ( $>100 \mathrm{ps})$, the electron and hole momentum distributions, and hence the inequivalent strength of bonds of different orientation, relax on fs timescales. The driving force for the $\mathrm{T}_{2 \mathrm{z}}$ phonon will therefore be very short-lived and "impulsive" in character. The rise of the SC field is limited by the drift of carriers within the built-in potential, and so the excitation of the COP will be delayed. The form of the data plotted in the Argand plane in Figure 2(f) can now be understood. When $\varphi=0$ and $90^{\circ}$, the amplitude of the COP excited by ISRS is expected to vanish, revealing the amplitude and phase of excitation due to the SC field, which is represented by the vector $S_{\mathrm{SC}}$. As $\varphi$ is varied, the amplitude of the phonon excited by ISRS varies, but with constant phase, tracing out a straight line that passes through the origin. The net effect of ISRS and SC field excitation is a straight line, offset from the origin. It is straightforward to fit a straight line to the data within the Argand plane, and hence generate the curves shown in Figure 2 (d-e) (see Supplementary Information S6).

The COPs observed in the transient AR response of $\mathrm{Ge}_{2} \mathrm{Sb}_{2} \mathrm{Te}_{5} /$ $\mathrm{GaSb}(001)$ were analyzed in a similar manner. Fitting yielded two oscillatory components superimposed on a complicated background. The frequency and relaxation time of the first COP of larger 

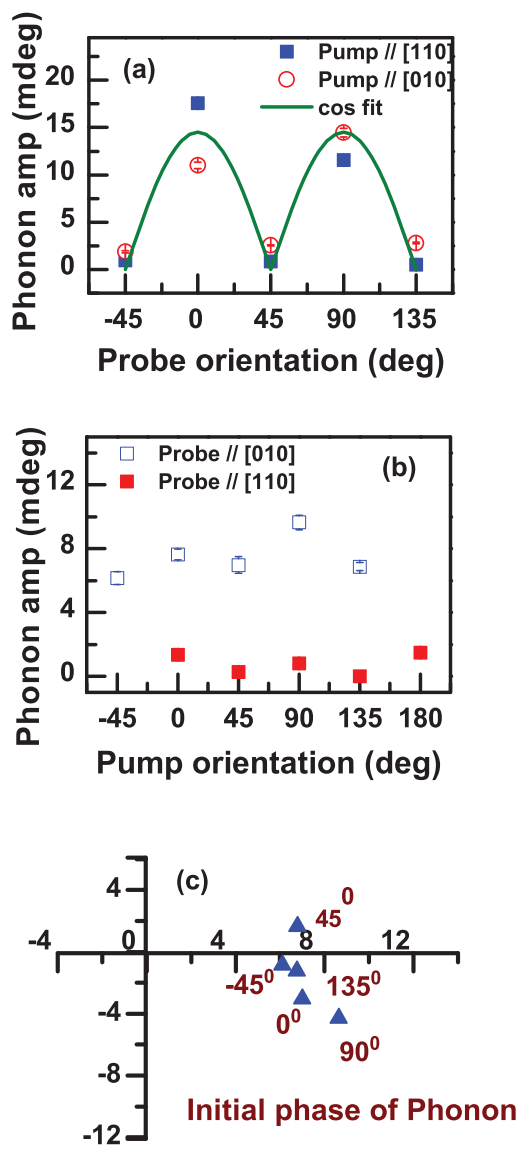

Figure $3 \mid$ Dependence of the amplitude and phase of the $3.4 \mathrm{THz}$ mode observed in $\mathrm{Ge}_{2} \mathrm{Sb}_{2} \mathrm{Te}_{5} / \mathrm{GaSb}(001)$ upon (a) probe and (b) pump polarization. (c) The dependence of amplitude and phase upon the pump polarization are plotted in the Argand plane.

amplitude were $3.4 \pm 0.1 \mathrm{THz}$ and 0.5 ps respectively, and $6.79 \pm$ $0.02 \mathrm{THz}$ and 3.0 ps respectively for the second COP. The frequencies were again found to be independent of the pump and probe polarization (see Supplementary Information S7). The $6.79 \mathrm{THz}$ COP can again be attributed to the zone centre TO phonon of the GaSb homoepitaxial layer and substrate, while the $3.4 \mathrm{THz}$ COP originates in the $\mathrm{Ge}_{2} \mathrm{Sb}_{2} \mathrm{Te}_{5}$ layer. The variation of the amplitude and phase of the $6.79 \mathrm{THz} \mathrm{COP}$ is consistent with that in Fig. 2 although less clear due to the smaller signal amplitude (see Supplementary Information S7). Fig. 3(a) and (b) show the dependence of the amplitude of the $3.4 \mathrm{THz} \mathrm{COP}$ upon the probe and pump polarization respectively. While the dependence upon probe polarization is similar to that observed in Fig. 2(c), there is only a weak dependence upon pump polarization. The data is plotted in the Argand plane in Figure 3(c). The clustering of data points close to the horizontal axis indicates that the phase of the COP is also insensitive to the pump polarization. Further plots of the phase of the $3.4 \mathrm{THz} \mathrm{COP}$, and the amplitude of the $6.77 \mathrm{THz} \mathrm{COP}$ and the longer-lived transient in the AR signal are presented in the Supplementary Information S7.

The epitaxial $\mathrm{Ge}_{2} \mathrm{Sb}_{2} \mathrm{Te}_{5}$ film exhibits a distorted rock-salt structure with about $20 \%$ of the $\mathrm{Ge} / \mathrm{Sb}$ atomic sites being vacant. The ideal rock-salt structure has $\mathrm{O}_{\mathrm{h}}$ symmetry with five irreducible representations, usually denoted as $A_{1 \mathrm{~g}}, A_{2 \mathrm{~g}}, E_{g}, T_{1 \mathrm{~g}}$ and $T_{2 \mathrm{~g}}{ }^{26}$ but none of the associated phonon modes are Raman active ${ }^{26,27}$. As for zincblende, the threefold $\mathrm{T}_{2}$ mode is split into a LO phonon and doubly degenerate TO phonons at the zone centre, the LO mode having slightly higher frequency ${ }^{32}$. The Raman tensor for the $T_{2}$ mode has the same form for structures of both $\mathrm{O}_{\mathrm{h}}$ and $\mathrm{T}_{\mathrm{d}}$ symmetry. However, the structure of $\mathrm{Ge}_{2} \mathrm{Sb}_{2} \mathrm{Te}_{5}$ is only 'rock salt like'. Ordering of vacancies and the displacement of ions from their positions in the ideal rock salt structure may remove the inversion symmetry, allowing $1^{\text {st }}$ order Raman scattering and also excitation of COPs by a SC field. Indeed the character of the allowed phonon modes may be modified. For example the zone-center $\mathrm{T}_{2}$ mode might be transformed into 1-D A and 2-D E modes if the symmetry of the crystal is reduced.

In previous studies of amorphous and polycrystalline $\mathrm{Ge}_{2} \mathrm{Sb}_{2} \mathrm{Te}_{5}$ films, a $3.4 \mathrm{THz}$ mode observed in the $\mathrm{R}$ signal was assigned $\mathrm{A}_{1 \mathrm{~g}}$ character $^{5-8}$. However, Merlin's theory (described in Supplementary Information S4) predicts that the A and E modes should be observed only within the $\mathrm{R}$ signal, with no dependence on pump or probe polarization, while being absent from the AR signal. The weak oscillations at $4.2-4.5 \mathrm{THz}$ in the $\mathrm{R}$ signal in Fig. 1(a) have frequency close to those observed previously ${ }^{5,6,9}$ and are attributed to an A mode. However, the observation of the $3.4 \mathrm{THz}$ mode in the AR signal, and its dependence on probe polarization, point to $\mathrm{T}_{2}$-like character. Its frequency suggests that $\mathrm{Sb}$-Te bonds play an important role ${ }^{5}$. In contrast to the $6.77 \mathrm{THz} \mathrm{T}_{2 \mathrm{z}}$ mode of $\mathrm{GaSb}$, the amplitude and phase of the $\mathrm{T}_{2}$-like mode in $\mathrm{Ge}_{2} \mathrm{Sb}_{2} \mathrm{Te}_{5}$ have only a weak and unclear dependence on the pump polarization. This suggests that the COP is excited by a SC field, with negligible contribution from ISRS. Excitation may occur within the $\mathrm{Ge}_{2} \mathrm{Sb}_{2} \mathrm{Te}_{5}$ if broken inversion symmetry causes the $\mathrm{Ge}_{2} \mathrm{Sb}_{2} \mathrm{Te}_{5}$ to become piezoelectric. Alternatively the response of the GaSb to a SC field may generate impulsive excitation of the $\mathrm{Ge}_{2} \mathrm{Sb}_{2} \mathrm{Te}_{5}$ at the $\mathrm{GaSb} / \mathrm{Ge}_{2} \mathrm{Sb}_{2} \mathrm{Te}_{5}$ interface.

Previous studies ${ }^{31,33-35}$ have shown that structural phase transitions can be induced in $\mathrm{Ge}_{2} \mathrm{Sb}_{2} \mathrm{Te}_{5}$ by single or multiple fs optical pulses. Here the dependence of the R and AR signals and the associated COP upon pump fluence was investigated with pump and probe electric fields parallel to the [110] and [100] axes respectively. A measurement was made on as-deposited material at elevated pump fluence and then immediately repeated with a reduced fluence of $0.42 \mathrm{~mJ} / \mathrm{cm}^{2}$. The power spectra calculated from the repeated scans
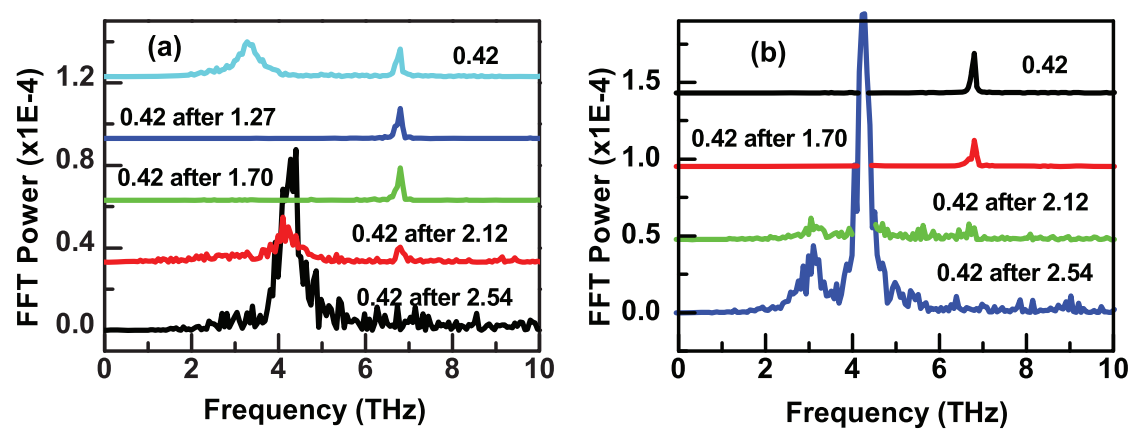

Figure 4 | Power spectra obtained from AR signals measured with a pump fluence of $0.42 \mathrm{~mJ} / \mathrm{cm}^{2}$, after exposure of as-deposited (a) $\mathrm{Ge}_{2} \mathrm{Sb}_{2} \mathrm{Te}_{5} / \mathrm{GaSb}$ and (b) GaSb samples to the higher pump fluences indicated in the plot. 
on $\mathrm{Ge}_{2} \mathrm{Sb}_{2} \mathrm{Te}_{5} / \mathrm{GaSb}$ and $\mathrm{GaSb}$ are shown in Fig. 4(a) and (b) respectively. The scans performed at the elevated fluence (not shown) exhibited similar behavior for both $\mathrm{Ge}_{2} \mathrm{Sb}_{2} \mathrm{Te}_{5} / \mathrm{GaSb}$ and $\mathrm{GaSb}$. For small fluences the amplitudes of the R and AR signals increased linearly with pump fluence. COPs were clearly observed within the AR signal. While their amplitude increased linearly with pump fluence, their frequency and relaxation time were observed to decrease. For $\mathrm{Ge}_{2} \mathrm{Sb}_{2} \mathrm{Te}_{5} / \mathrm{GaSb}$ the relaxation time of the $3.4 \mathrm{THz}$ mode decreased linearly from 660 fs to $470 \mathrm{fs}$ as the fluence increased from 0.42 to $1.27 \mathrm{~mJ} / \mathrm{cm}^{2}$, while for $\mathrm{GaSb}$ the relaxation time of the $6.77 \mathrm{THz}$ mode decreased from 5.0 ps to 3.5 ps as the fluence increased from 0.42 to $1.70 \mathrm{~mJ} / \mathrm{cm}^{2}$. Further details are provided in supplementary information $\mathrm{S} 8$.

The repeated scans at $0.42 \mathrm{~mJ} / \mathrm{cm}^{2}$ fluence show that the $3.4 \mathrm{THz}$ mode in $\mathrm{Ge}_{2} \mathrm{Sb}_{2} \mathrm{Te}_{5}$ vanished after exposure to a fluence of $1.70 \mathrm{~mJ} /$ $\mathrm{cm}^{2}$, while the $6.77 \mathrm{THz}$ mode vanished after exposure to a fluence of $2.12 \mathrm{~mJ} / \mathrm{cm}^{2}$ in both samples. COPs instead appeared in the AR signal at frequencies of $4.2 \mathrm{THz}$ in both samples and at $3.1 \mathrm{THz}$ for the $\mathrm{Ge}_{2} \mathrm{Sb}_{2} \mathrm{Te}_{5} / \mathrm{GaSb}$ sample. The $4.2 \mathrm{THz}$ mode also appeared with large amplitude in the transient $\mathrm{R}$ signal (not shown). The 4.2 and 3.1 THz COPs may be attributed to the $A_{1 g}$ and $E_{g}$ modes of elemental $\mathrm{Sb}$, that segregates at the elevated temperatures induced by the pump ${ }^{7}$. The frequencies observed in the present study are about $0.4 \mathrm{THz}$ lower and hence distinct from those commonly observed by others in the $\mathrm{R}$ signal from amorphous and polycrystalline $\mathrm{Ge}_{2} \mathrm{Sb}_{2} \mathrm{Te}_{5}{ }^{21,36}$.

In summary, comprehensive time-resolved reflectance and anisotropic reflectance measurements have been performed upon epitaxial $\mathrm{Ge}_{2} \mathrm{Sb}_{2} \mathrm{Te}_{5} / \mathrm{GaSb}(001)$ and $\mathrm{GaSb}(001)$ samples. The orientation of pump and probe electric fields have been varied relative to the crystallographic axes. The AR measurements are richly featured and yield relaxation times that can be ascribed to the interaction of the different populations of electrons and phonons. $\mathrm{GaSb}(001)$ is an exemplar in which the observation of the $\mathrm{T}_{2}$ transverse optical phonon has been shown to result from a combination of ISRS and the action of a SC field. The results can be fully understood both in terms of a theory based upon use of the Raman tensor, and also from a microscopic model of selective optical bond breaking. Epitaxial $\mathrm{Ge}_{2} \mathrm{Sb}_{2} \mathrm{Te}_{5} / \mathrm{GaSb}(100)$ exhibits both the $\mathrm{T}_{2}$ mode supported by the substrate and also a $\mathrm{T}_{2}$-like mode associated with the $\mathrm{Ge}_{2} \mathrm{Sb}_{2} \mathrm{Te}_{5}$. The insensitivity to the pump polarization suggests that the $\mathrm{T}_{2}$-like mode is excited by a SC field, either in the $\mathrm{Ge}_{2} \mathrm{Sb}_{2} \mathrm{Te}_{5}$ if the distorted rock salt structure lacks inversion symmetry, or by a SC field in the GaSb leading to impulsive excitation of the $\mathrm{Ge}_{2} \mathrm{Sb}_{2} \mathrm{Te}_{5}$ at the $\mathrm{Ge}_{2} \mathrm{Sb}_{2} \mathrm{Te}_{5} / \mathrm{GaSb}$ interface. The observation of the $\mathrm{T}_{2}$-like mode provides confirmation that the $\mathrm{Ge}_{2} \mathrm{Sb}_{2} \mathrm{Te}_{5}$ is essentially cubic and challenges mode assignments made in previous studies. The SC field also generates lateral stress in the lattice that leads to a long-lived transient AR response. This may be important for the generation of surface acoustic waves (SAWs) that can be steered by means of the optical polarization, without the need for an overlaid grating. Finally, the formation of a new phase after exposure to large pump fluence was observed in both $\mathrm{Ge}_{2} \mathrm{Sb}_{2} \mathrm{Te}_{5}$ and $\mathrm{Ge}_{2} \mathrm{Sb}_{2} \mathrm{Te}_{5} / \mathrm{GaSb}$. The observed frequencies suggest segregation of $\mathrm{Sb}$, while increased disorder in the $\mathrm{GaSb}$ and $\mathrm{Ge}_{2} \mathrm{Sb}_{2} \mathrm{Te}_{5}$ leads to the disappearance of the $\mathrm{T}_{2}$ and $\mathrm{T}_{2}$-like modes from the AR signal.

\section{Methods}

Time-resolved $\mathrm{R}$ and AR (rotation) measurements were performed upon a $\mathrm{Ge}_{2} \mathrm{Sb}_{2} \mathrm{Te}_{5}(15 \mathrm{~nm}) / \mathrm{GaSb}(50-100 \mathrm{~nm}) / \mathrm{GaSb}(001)$ structure prepared by molecular beam epitaxy. The $\mathrm{Ge}_{2} \mathrm{Sb}_{2} \mathrm{Te}_{5}$ exhibits a predominant cube on cube growth with the $\mathrm{Ge}_{2} \mathrm{Sb}_{2} \mathrm{Te}_{5}[100]$ axis parallel to the $\mathrm{GaSb}[100]$ axis $^{10}$. Additional measurements were performed upon a reference structure without the $\mathrm{Ge}_{2} \mathrm{Sb}_{2} \mathrm{Te}_{5}$ layer. Pump and probe pulses of $800 \mathrm{~nm}$ wavelength and $55 \mathrm{fs}$ duration were generated by a Ti:sapphire regenerative amplifier system at $100 \mathrm{kHz}$ repetition rate. The linearly polarized pump pulse with fluence of $0.85 \mathrm{~mJ} / \mathrm{cm}^{2}$ was directed onto the sample at close to normal incidence. The $\mathrm{R}$ and $\mathrm{AR}$ response were measured by a time delayed $s$-polarized probe pulse with fluence of $0.2 \mathrm{~mJ} / \mathrm{cm}^{2}$ incident at $45^{\circ}$ to the sample normal. An optical chopper was placed in the pump beam so that phase sensitive detection could be used to extract the transient R and AR signals (see Supplementary Information: S9). The polarization of the pump and probe beams relative to the crystallographic axes of the samples was controlled by rotating the sample about its normal, and by rotating a polarizer placed in the path of the pump beam.

1. Wuttig, M. \& Yamada, N. Phase-change materials for rewriteable data storage. Nature Mater. 6, 824-832 (2007).

2. Lankhorst, M. H. R., Ketelaars, B. W. S. M. M. \& Wolters, R. A. M. Low-cost and nanoscale non-volatile memory concept for future silicon chips. Nature Mater. 4, 347-352 (2005).

3. Wright, C. D., Liu, Y., Kohary, K. I., Aziz, M. M. \& Hicken, R. J. Arithmetic and biologically-inspired computing using phase-change materials. Adv. Mater. 23, 3408-3413 (2011).

4. Wright, C. D., Hosseini, P. \& Diosdado, J. A. V. Beyond von-Neumann Computing with Nanoscale Phase-Change Memory Devices. Adv. Funct. Mater. 23, 2248-2254 (2013).

5. Först, M., Dekorsy, T., Trappe, C., Laurenzis, M. \& Kurz, H. Phase change in $\mathrm{Ge}_{2} \mathrm{Sb}_{2} \mathrm{Te}_{5}$ films investigated by coherent phonon spectroscopy. Appl. Phys. Lett. 77, 1964-1966 (2000).

6. Hase, M., Miyamoto, Y. \& Tominaga, J. Ultrafast dephasing of coherent optical phonons in atomically controlled $\mathrm{GeTe} / \mathrm{Sb}_{2} \mathrm{Te}_{3}$ superlattices. Phys. Rev. B 79, 174112 (2009).

7. Li, Y., Stoica, V. A., Endicott, L., Wang, G. \& Uher, C. Coherent optical phonon spectroscopy studies of femtosecond-laser modified $\mathrm{Sb}_{2} \mathrm{Te}_{3}$ films. Appl. Phys. Lett. 97, 171908 (2010).

8. Makino, K., Tominaga, J. \& Hase, M. Ultrafast optical manipulation of atomic arrangements in chalcogenide alloy memory materials. Opt. Express. 19, 1260-1270 (2011).

9. Savoia, A., Gawelda, W., Solis, J. \& Mansart, B. Coherent optical phonons in different phases of $\mathrm{Ge}_{2} \mathrm{Sb}_{2} \mathrm{Te}_{5}$ upon strong laser excitation. Appl. Phys. Lett. $\mathbf{9 8}$ 251906 (2011).

10. Braun, W. et al. Epitaxy of Ge-Sb-Te phase-change memory alloys. Appl. Phys. Lett. 94, 041902 (2009).

11. Merlin, R. Generating coherent $\mathrm{THz}$ phonons with light pulses. Solid State Commun. 102, 207-220 (1997).

12. Dekorsy, T., Cho, G. C. \& Kurz, H. Coherent phonons in condensed media. Light Scattering in Solids 76, 169-209 (2000).

13. Cho, G. C., Kütt, W. \& Kurz, H. Subpicosecond Time-resolved Coherent-Phonon Oscillations in GaAs. Phys. Rev. Lett. 65, 764-766 (1990).

14. Qi, J. et al. Ultrafast carrier and phonon dynamics in $\mathrm{Bi}_{2} \mathrm{Se}_{3}$ crystals. Appl. Phys. Lett. 97, 182102 (2010).

15. Pelouch, W. S. \& Schlie, L. A. Ultrafast carrier dynamics in GaSb. Appl. Phys. Lett. 66, 82-84 (1995).

16. Wright, O. B., Zammit, U., Marinelli, M. \& Gusev, V. E. Picosecond relaxation and thermal diffusion in amorphous silicon. Appl. Phys. Lett. 69, 553 (1996).

17. Sabbah, A. \& Riffe, D. Femtosecond pump-probe reflectivity study of silicon carrier dynamics. Phys. Rev. B 66, 165217 (2002).

18. Wilks, R., Hughes, N. D. \& Hicken, R. J. Investigation of transient linear and circular birefringence in metallic thin films. J. Phys.: Condens. Matter 15, 5129-5143 (2003).

19. Wilks, R. \& Hicken, R. J. Transient optical polarization response of aluminium at an interband transition. J. Physics.: Condens. Matter 16, 4607-4617 (2004).

20. Sundaram, S. K. \& Mazur, E. Inducing and probing non-thermal transitions in semiconductors using femtosecond laser pulses. Nat. Mater. 1, 217-224 (2002).

21. Garrett, G., Albrecht, T., Whitaker, J. \& Merlin, R. Coherent THz Phonons Driven by Light Pulses and the Sb Problem: What is the Mechanism? Phys. Rev. Lett. 77, 3661-3664 (1996).

22. Miller, J. et al. Near-bandgap wavelength dependence of long-lived traveling coherent longitudinal acoustic phonons in GaSb-GaAs heterostructures. Phys. Rev. B 74, 113313 (2006).

23. Pfeifer, T., Kütt, W. \& Kurz, H. Generation and Detection of Coherent Optical Phonons in Germanium. Phys. Rev. Lett. 69, 3248-3251 (1992).

24. Dekorsy, T., Pfeifer, T., Kütt, W. \& Kurz, H. Subpicosecond carrier transport in GaAs surface-space-charge fields. Phys. Rev. B 47, 3842-3849 (1993).

25. Yee, K., Lee, K., Oh, E., Kim, D. \& Lim, Y. Coherent Optical Phonon Oscillations in Bulk GaN Excited by Far below the Band Gap Photons. Phys. Rev. Lett. 88, 105501 (2002).

26. Cardona, M. \& Güntherodt, G. Topics in applied physics: Light Scattering in Solids II. Optics \& Laser Technology 9, (Springer-Verlag New York, 1982).

27. Hayes, W. \& Loudon, R. Scattering of light by crystals. Advances in Physics (Dover publications New York, 2004).

28. Yu, P. Y. \& Cardona, M. Fundamentals of Semiconductors: Physics and Materials Properties (Springer-Verlag Berlin Heidelberg, 2010).

29. Ferrini, R. et al. Phonon response of $\mathrm{Al}_{\mathrm{x}} \mathrm{Ga}_{1-\mathrm{x}} \mathrm{Sb} / \mathrm{GaSb}$ epitaxial layers by Fouriertransform infrared-reflectance and Raman spectroscopies. Phys. Rev. B 56, 7549-7553 (1997).

30. Farr, M. K., Traylor, J. G. \& Sinha, S. K. Lattice dynamics of GaSb. Phys. Rev. B 11, 1587-1594 (1975).

31. Urbanowicz, A., Adomavic, R. \& Krotkus, A. Terahertz emission from photoexcited surfaces of Ge crystals. Physica B 367, 152-157 (2005). 
32. Decius, J. C. \& Hexter, R. M. Molecular vibrations in crystals. (McGraw-Hill, 1970).

33. Liu, Y., Aziz, M. M., Shalini, A., Wright, C. D. \& Hicken, R. J. Crystallization of $\mathrm{Ge}_{2} \mathrm{Sb}_{2} \mathrm{Te}_{5}$ films by amplified femtosecond optical pulses. J. Appl. Phys. 112, 123526 (2012)

34. Siegel, J., Gawelda, W., Puerto, D., Dorronsoro, C. \& Solis, J. Amorphization dynamics of $\mathrm{Ge}_{2} \mathrm{Sb}_{2} \mathrm{Te}_{5}$ films upon nano- and femtosecond laser pulse irradiation. J. Appl. Phys. 103, 023516 (2008).

35. Cotton, R. L. \& Siegel, J. Stimulated crystallization of melt-quenched $\mathrm{Ge}_{2} \mathrm{Sb}_{2} \mathrm{Te}_{5}$ films employing femtosecond laser double pulses. J. Appl. Phys. 112, 123520 (2012).

36. Ishioka, K., Kitajima, M. \& Misochko, O. V. Coherent $A_{1 g}$ and $E_{g}$ phonons of antimony. J. Appl. Phys. 103, 123505 (2008).

\section{Acknowledgements}

The authors gratefully acknowledge financial support from Engineering and Physical Sciences Research Council grant EP/F015046/1.

\section{Author contributions}

F.K. and W.B. prepared the epitaxial $\mathrm{Ge}_{2} \mathrm{Sb}_{2} \mathrm{Te}_{5}(001)$ and $\mathrm{GaSb}(001)$ samples. A.S., U.A.S.A., Y.L. and R.J.H. developed the measurement procedure. A.S., U.A.S.A. and Y.L. made the pump-probe measurements, Y.L. performed the fitting of the data, and A.S. prepared the figures. R.J.H. developed the microscopic and Raman tensor based models. G.P.S., C.D.W. and R.J.H. developed the interpretation of the data. R.J.H. conceived the project, while Y.L., A.S. and R.J.H. together wrote the paper.

\section{Additional information}

Supplementary information accompanies this paper at http://www.nature.com/ scientificreports

Further underlying research materials may be found at http://hdl.handle.net/10871/13760 Competing financial interests: The authors declare no competing financial interests. How to cite this article: Shalini, A. et al. Observation of $\mathrm{T}_{2}$-like coherent optical phonons in epitaxial $\mathrm{Ge}_{2} \mathrm{Sb}_{2} \mathrm{Te}_{5} / \mathrm{GaSb}(001)$ films. Sci. Rep. 3, 2965; DOI:10.1038/srep02965 (2013). 